\title{
Engineering Antibody Fragment with the Quantum Dot in Cancer Cell Imaging and Diagnosis
}

\author{
Lizhi Liu, Weiming $\mathrm{Xu}^{*}$ \\ Krebs Institute, Department of Molecular Biology and Biotechnology, University of Sheffield, Western Bank, Firth Court, Sheffield, UK \\ Email: *Weiming.Xu@Sheffield.ac.uk
}

Received 2012

\begin{abstract}
The conjugates of monoclonal antibodies and nanoparticles, including quantum-dot(QD), offer significant advantages over conventional fluorescent probes to image and study biological processes. The extend stability, intense fluorescence and low toxicity of QDs are well suited for biological applications. In the present study, we used QD-conjugated anti-glucose-regulated protein 78(GRP78) antibody to examine the gene expression in prostate cancer cells under the unfolded protein response (UPR). QDs have got unique, simple and fast properties over current diagnostic techniques such as peroxidase-based immunohistochemical staining procedures, therefore the nanocarrier-conjugated antibody fragment has potential to become a new therapeutic tool for cancer diagnosis and treatment.
\end{abstract}

Keywords: Quantum Dot; Antibody Fragment; Prostate Cancer; GRP78; scFv; UPR; Immunohistochemistry

\section{Introduction}

Recent development on semiconductor nanocrystal quantum dots (QDs), has hold particular new promise as next generation of fluorescent probes[1,2]. The nanoparticles, such as cadmium selenide quantum dots, only 100-100,000 atoms in size, can emit intrinsic fluorescence often many times stronger than that observed for other classes of fluorophores. In the traditional engineering fields, QDs are developing as components of solar cells, quantum computers, light-emitting diodes and others. Recently, in the bioengineering filed, increasing number of biological scientists, are employing such QDs for use in image-guided surgery, light-activated therapies and biomarker development[3]. To further explore the nanotechnology in cancer diagnosis and treatment, we chose a specific cancer biomarker, glucose-regulated protein 78(GRP78), as our target biomarker. GRP78 is a master switch in unfold protein response. It plays a crucial role in cancer cell proliferation, angiogenesis and chemotherapy drug resistance[4,5]. Especially, In double knock-out PTEN (a tumor suppressor cells) and GRP78, prostate tumorigenesis was[6] blocked and prostate cancer development were completely halted, indicating GRP78 being a crucial gene in prostate cancer growth, cell invasion and angiogenesis. In another research, another tumor suppressor gene, prostate apoptosis response-4 (Par-4), induced cancer cell apoptosis by binding the GRP78 on cell surface[7].

Previously we reported that nitric oxide induced coupling mitochondria respiration to ER-stress, inducing GRP78 expression[8]. Furthermore, we have targeted membrane-bound GRP78 by subtractive screening of single chain variable fragment (scFv) library and successful isolated a panel of scFv specific for GRP78[9]. Recently, we have performed proof-ofconcept studies investigating the therapeutic potential of a hu-

${ }^{*}$ Corresponding author. manized scFv- GRP78 antibody in nude mouse model. We labeled the antibody with quantum dot-(QD) nanobeads and tested its biological function in xenograft breast cancer nude mouse model and found the Qdot- conjugated anti- GRP78 $\mathrm{scFv}$ inhibits cancer growth in mice[10].To further explore the nanotechnology in cancer diagnosis and treatment, we apply the QD-conjugated GRP78 antibody to detect the GRP78 expression in prostate cancer cell in tissue culture condition and in the formalin fixed paraffin embedded (FFPE) cancer cell slides.

\section{Experimental Procedures}

\subsection{Cell Culture}

The human prostate cancer cell line LNCaP was from the European Cell Bank (ECACC). Cells were grown in DMEM medium with $10 \%$ fetal calf serum in $37^{\circ} \mathrm{C}$ incubator with $5 \%$ CO2. For induce the UPR response, the cells were treated with $200 \mathrm{nM}$ thapsigargin(TG) for $12 \mathrm{~h}$.

\subsection{QD Labeling of Anti-GRP78 scFv Antibody}

ScFv-Grp78-H19 antibody was conjugated with a Qdot-625 Antibody Conjugation Kits (Cat A10197, Invitrogen, UK) as previously described[10]. Briefly, Qdot-625 beads were purchased from the Invitrogen. The beads were activated through crosslinker 4-(maleimidomethyl)-1-cyclohexanecarboxylic acid $\mathrm{N}$ hydroxysuccinimide ester (SMCC). The thiol containing antibody reduced by DTT were covalently coupled with activated Qdot-625 nanocrystals. The conjugated QD-antibody was purified through ultrafiltration and size exclusion chromatography. The specificity of the conjugated antibody was confirmed by dot blot and western blot as described previously[10].

\subsection{QDs Fluorescence Immunohistochemistry}

LNCaP human prostate cancer cells were washed with PBS and 
permeabilized in PBS containing 0.1\% Triton X-100 and 1\% horse serum for $60 \mathrm{~min}$. The cells fixed with $4 \%$ paraformaldehyde for $30 \mathrm{~min}$. Cells were prehybridized with PBS containing $0.1 \%$ Triton $\mathrm{X}-100$ and $1 \%$ horse serum for $2 \mathrm{~h}$ and then stained directly with $10 \mathrm{nM}$ Qot-GRP78 antibody diluted with PBS containing $0.1 \%$ Triton $\mathrm{X}-100$ and $1 \%$ horse serum overnight at $4^{0} \mathrm{C}$. After washing with PBS, the cells were directly observed under the on a confocal microscope (Leica TCS SP, Germany) with $470 \mathrm{~nm}$ excitation and $624 \mathrm{~nm}$ emission filters. The $10 \mathrm{nM}$ unconjugated Qdot 625 beads were used as a control.

\subsection{In situ Immunohistochmistry by QDs in the Formalin Fixed Paraffin Embedded (FFPE) Cancer Cell Slides}

LNCaP human prostate cancer cells in formalin fixed paraffin embedded (FFPE) cancer cell blocks were dewaxed and the antigen was retrieved using $1 \%$ SDS in TBS buffer (100 mM Tris $\mathrm{pH} 7.4,138 \mathrm{mM} \mathrm{NaCl}, 27 \mathrm{mM} \mathrm{KCl}$ ). The sections were then prehybridized with PBS containing $0.1 \%$ Triton X-100 and $1 \%$ horse serum for $2 \mathrm{~h}$ and then stained directly with $10 \mathrm{nM}$ Qot-GRP78 antibody diluted with PBS containing $0.1 \%$ Triton $\mathrm{X}-100$ and $1 \%$ horse serum overnight at $4^{\circ} \mathrm{C}$. After washing with PBS, the cells were visualized using a Nikon Eclipase E400 fluorescence microscope with the $365 \mathrm{~nm}$ excitation filter and FITC emission filter. For direct comparison with the conventional immunohistochemistry, the same series sections were blocking endogenous peroxidase activity with $3 \% \mathrm{H}_{2} \mathrm{O}_{2}$ for 15 min, the antigen was retrieved using $1 \%$ SDS in TBS buffer (100 mM Tris pH 7.4, $138 \mathrm{mM} \mathrm{NaCl,} 27 \mathrm{mM} \mathrm{KCl}$ ) were prehybridized with PBS containing $0.1 \%$ Triton X-100 and $1 \%$ horse serum for $2 \mathrm{~h}$ and then cells were stained with a 1:1,000 dilution of anti- GRP78(Monoclonal antibody, BD) and the second antibody was the biotin-conjugated anti-mouse IgG. Then Vecta-stain 'ABC kit' (Vector Laboratories) was used with indirect avidin-biotin horseradish peroxidase staining with Diaminiobenzidine (DAB) as substrate.

\section{Results and Discussion}

\subsection{Detection of UPR Response Gene GRP78 in Prostate Cancer Cells by QD-anti-GRP78 Antibody}

The endoplasmic reticulum (ER) is a perinuclear organelle that is responsible for protein synthesis and folding. When the folding capacity of the ER is exceeded, the unfolded protein response (UPR) is triggered. Cancer cells have the ability to grow very rapidly, often ahead from their blood supply. This causes a harsh environment where hypoxia, acidity and glucose starvation occurs. This situation leads to the formation of UPR and ER stress. Grp78 plays a central role in the UPR. Many ER stress inducers, including thapsigargin, MG132 or paclitaxel, increased expression levels of GRP78 in cancer[11]. In this study, we used 200nM TG to treat LNCaP human prostate cancer cells for 12h and stained with 10nM Qdot-GRP78 antibody overnight. As shown on Figure 1B, TG treated prostate cancer cell express significant high level of GRP78 than that of untreated cells (Figure 1A). In the control group, there was no detectable GRP78 using the unconjugated Qdot 625 only (Fig- ure 1C).

\subsection{In situ Immunohistochmistry by Qdot in the Formalin Fixed Paraffin Embedded (FFPE) Cancer Cell Slides}

$5-\mu \mathrm{m}$ sections of paraffin-embedded formalin fixed prostate cancer LNCaP cells were stained using 30nM Qdot-GRP78 antibody overnight(Figure 2B). Figure 2A shows the phase contrast image of the consecutive slide. Figure 2B shows the bright red staining results from the Qdot-GRP78 staining. There was no background staining on the slide with unconjugated Qdot only (Figure 2C). In the control staining with the antiGRP78 monoclonal antibody, some of the prostate cancer cells were also GRP78 positive in peroxidase-based immunohistochemistry(Figure 2D).

In summary, we have shown that Qdot-labeled antibody can be used to study the gene expression in prostate cancer cells under the unfolded protein response(UPR). QDs have got unique, simple and fast properties over current diagnostic techniques such as peroxidase-based immunohistochemical staining procedures. In the conventional methods, there are 5 extra steps
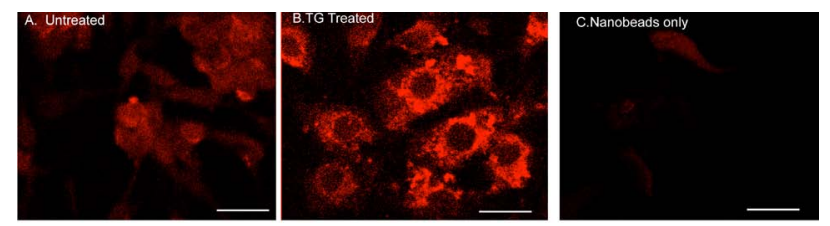

Figure 1. Quantum-Dot images of Qdot625-GRP78 staining of human LNCaP prostate cancer cells. A. untreated cell stained by Qdot625-GRP78. B. TG treated, upregulated GRP78, detected by Qdot625-GRP78. C. TG treated, stained with unconjugated Qdot625. Scale bar represents $20 \mu \mathrm{m}$.
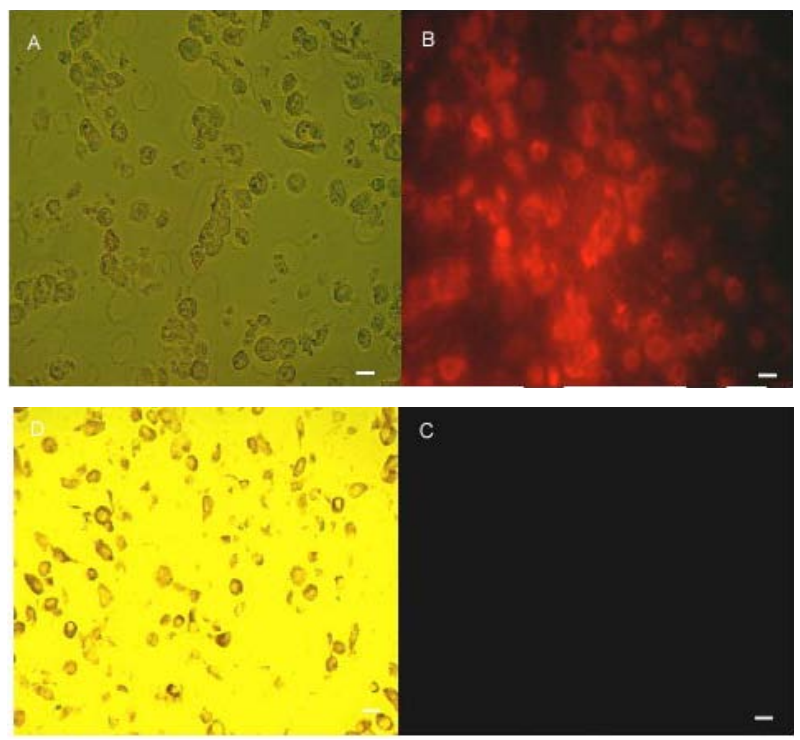

Figure 2. In situ Immunohistochmistry in the formalin fixed paraffin embedded (FFPE) cancer cells. A. phase contrast image of the LNCaP human prostate cancer cells. B. Quantum-Dot images of Qdot625- GRP78 staining of FFPE block. C. stained with unconjugated Qdot625. D. Conventional peroxidase-based immunohistochemical staining using the anti-GP78 mouse monoclonal antibody. Scale bar represents $20 \mu \mathrm{m}$. 


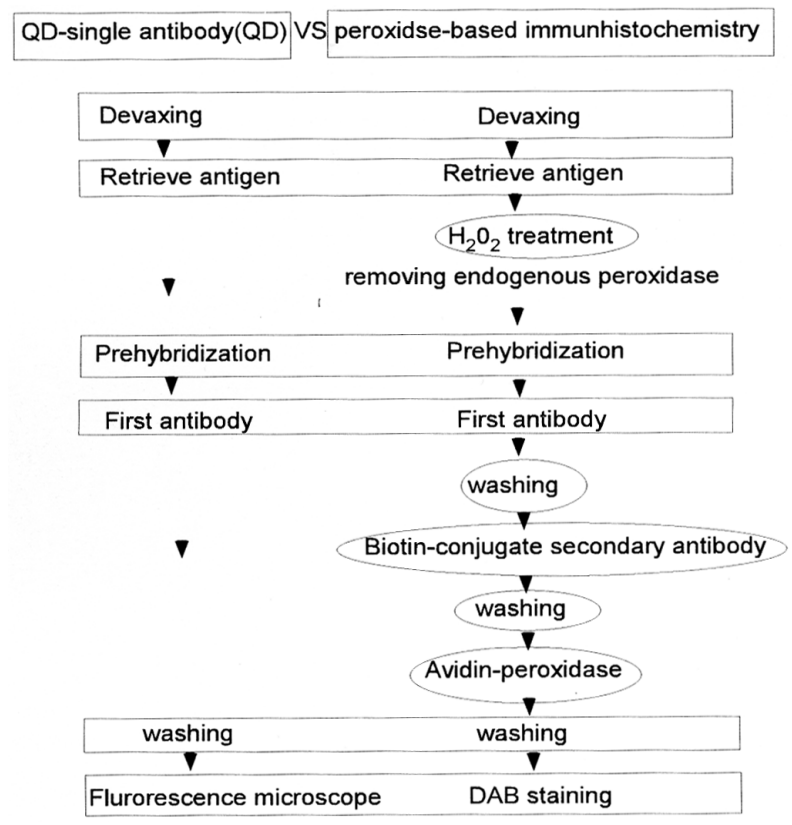

Figure 3. Comparison of QD staining and peroxidase-based immunohistochemistry.

(Figure 3) after devaxing; therefore nanocarrier-conjugated antibody fragment has potential to become a new early detection tool for cancer diagnosis. A recent pioneering study using primates for Qdots toxicity study has shown that its nanocrystals to be safe over a one-year period[12], therefore, QDs may have its potential for treatment of cancer in future.

\section{REFERENCES}

[1] X. Gao, Y. Cui, R. M. Levenson, L. W. Chung, S. Nie,” In vivo cancer targeting and imaging with semiconductor quantum dots,” Nat Biotechnol, Vol 22, No.8, 2004, pp. 969 -976.

[2] C. Yang, C. Xu, X. Wang, X. Hu, "Quantum dot-based biosensor for simultaneous detection of biomarker and therapeutic drug: first steps toward an assay for quantitative pharmacology, " Analyst, Vol. 137, No.5, 2012, pp.1205-1209.
[3] H. Tada, H. Higuchi, T. M. Wanatabe, N. Ohuchi, "In vivo real-time tracking of single quantum dots conjugated with monoclonal anti- HER2 antibody in tumors of mice," Cancer Res, Vol. 67, No. 3, 2007, pp.1138 -1144.

[4] A. S. Lee, "GRP78 induction in cancer: therapeutic and prognostic implications,” Cancer Res, Vol. 67, No. 8, 2007, pp. 34963499.

[5] K. L. Cook, A. N. Shajahan, A. Warri, L. Jin, L. A. Hilakivi-Clarke, R. Clarke,” Glucose- Regulated Protein 78 Controls Cross-talk between Apoptosis and Autophagy to Determine Antiestrogen Responsiveness,” Cancer Res, Vol. 72, No. 13, 2012, pp. 3337-3349.

[6] Y. Fu, S. Wey, M. Wang, R. Ye, C. P. Liao, P. Roy-Burman, A. S. Lee," Pten null prostate tumorigenesis and AKT activation are blocked by targeted knockout of ER chaperone GRP78/BiP in prostate epithelium,” Proc Natl Acad Sci U S A Vol. 105, No. 49, 2008, pp. 19444-19449.

[7] R. Burikhanov, Y. Zhao, A. Goswami, S. Qiu, S. R. Schwarze, V. M. Rangnekar," The tumor suppressor Par-4 activates an extrinsic pathwayfor apoptosis,” Cell, Vol. 138, No. 2, 2009, pp.377-388.

[8] W. Xu, L. Liu, I. G. Charles, S. Moncada, "Nitric oxide induces coupling of mitochondrial signalling with the endoplasmic reticulum stress response," Nat Cell Biol, Vol. 6, No. 11, 2004, pp.1129-1134.

[9] L. Liu, W. Xu, "Targeting Nitric Oxide Mediated Upregulation of Membrane-bound Glucose Regulated-protein 78 by Subtractive Single Chain Variable Fragment (scFv) Phage Display,” American Journal of Biomedical Sciences, Vol. 1, No. 4, 2009, pp. 321-325.

[10] W. Xu, L. Liu, N. J. Brown, S. Christian, D. Hornby,” Quantum dot-conjugated anti- GRP78 scFv inhibits cancer growth in mice,” Molecules, Vol. 17, No. 1, 2012, pp. 796-808.

[11] D. Dong, C. Stapleton, B. Luo, S. Xiong, W. Ye, Y. Zhang, N. Jhaveri, G. Zhu, R. Ye, Z. Liu, K. W. Bruhn, N. Craft, S. Groshen, F. M. Hofman, A. S. Lee, “A critical role for GRP78/BiP in the tumor microenvironment for neovascularization during tumor growth and Metastasis," Cancer Res, Vol. 71, No. 8, 2011, pp. 2848-2957.

[12] L. Ye, K. T. Yong, L. Liu, I. Roy, R. Hu, J. Zhu, H. Cai, W. C. Law, J. Liu, K. Wang, J. Liu, Y. Liu, Y. Hu, X. Zhang, M. T. Swihart, P.N. Prasad, "A pilot study in non-human primates shows no adverse response to intravenous injection of quantum dots,” Nat Nanotechnol, Vol. 7, No. 7, 2012, pp. 453-458. 